\title{
Recurrent Ovarian Adenocarcinoma
}

National Cancer Institute

\section{Source}

National Cancer Institute. Recurrent Ovarian Adenocarcinoma. NCI Thesaurus. Code C153614.

The reemergence of ovarian adenocarcinoma after a period of remission. 